\title{
Needs and preferences of patients regarding atopic dermatitis care in the era of new therapeutic options: a qualitative study
}

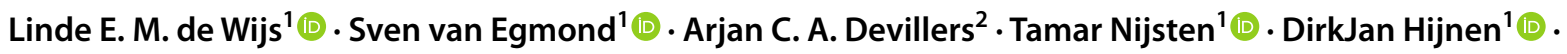 \\ Marjolein Lugtenberg ${ }^{1} \mathbb{D}$
}

Received: 18 May 2021 / Revised: 16 December 2021 / Accepted: 22 December 2021 / Published online: 2 February 2022

(c) The Author(s) 2022

\begin{abstract}
To optimally tailor atopic dermatitis (AD) care to patients' needs, especially considering the many emerging therapeutic options, insight into patients' needs and preferences regarding AD care is needed. To explore patients' needs and preferences regarding $\mathrm{AD}$ care, a qualitative study consisting of three focus groups with a total of 20 adult $\mathrm{AD}$ patients was conducted. All sessions were transcribed verbatim and inductively analyzed using several phases of coding to create an overview of patients' needs and preferences. AD patients emphasized the need for a patient-tailored approach in all identified aspects of AD care. With regard to consultations, patients stressed the need for a personal approach and increased recognition of the disease impact, which should mainly be determined by patients. With regard to the organization of AD care, the need for psychosocial and medical supportive care as well as quick access to health-care providers during disease flares was emphasized. Within the decision-making process, patients indicated that the provided information, the role of the patient and physician, whether or not treatment goals should be set, and decisive factors for indication and feasibility of novel therapies should be patient dependent. AD care should be patient tailored with increased attention for the psychosocial burden, as well as better access to health care during disease flares. To provide patient-tailored care, the personal situation, needs, and preferences of the patient should be taken into account in the therapeutic decision-making process, with respect for the autonomy of the patient.
\end{abstract}

Keywords Atopic dermatitis · Atopic eczema $\cdot$ Therapy $\cdot$ Qualitative research $\cdot$ Patient-centered care

\section{Introduction}

Atopic dermatitis (AD) is a chronic inflammatory skin disease, characterized by a relapsing and remitting disease course. AD-related symptoms including itch, pain and insomnia are known to have a large impact on quality of life [1-4]. Better acceptance and recognition of the emotional and psychological burden associated with $\mathrm{AD}$ are among the most important wishes of AD patients [3].

Linde E. M. de Wijs

1.dewijs@erasmusmc.nl

$\checkmark$ DirkJan Hijnen

d.hijnen@erasmusmc.nl

1 Department of Dermatology, Erasmus MC University Medical Center, Dr. Molewaterplein 40, 3015 GD Rotterdam, The Netherlands

2 Department of Dermatology, Maasstad Hospital, Rotterdam, The Netherlands
AD treatment is mainly focused on medicinal therapy, usually starting with topical treatment including emollients, topical corticosteroids, and calcineurin inhibitors [5]. Until recently, treatment of patients with moderate-to-severe $\mathrm{AD}$ was limited to phototherapy or conventional systemic immunosuppressants. However, the number of therapeutic options available to physicians has increased rapidly in the last few years [6]. At the same time, patients' preferences, standards, and expectations are changing and play an increasing role in the therapeutic decision-making process [7, 8].

Experiences, needs, and preferences regarding specific topics (e.g., autonomy, influence of (social) media, influence on family life or financial impact) have been assessed, mainly among adolescents or parents of pediatric AD patients [1-3, 9-11]. However, a complete overview concerning all aspects of today's $\mathrm{AD}$ care is needed to adequately tailor $\mathrm{AD}$ care to patients' needs, especially in times of many emerging therapies. Therefore, the aim of this study was to explore patients' needs and preferences regarding AD care. 


\section{Methods}

A qualitative study consisting of three focus groups was conducted to obtain an in-depth understanding of patients' views on $\mathrm{AD}$ care. Qualitative research is particularly suitable as it primarily focuses on interpretations, rather than quantification of patients' beliefs, emotions, behaviors, and interactions in daily life [12]. Additionally, the interactive and dynamic process of focus groups attributes to identification and clarification of patients' views, leading to richer and more diverse information in comparison to individual interviews $[13,14]$. This focus group study was designed and is reported in accordance with the SRQR recommendations. [15].

\section{Study setting and participants}

This study was conducted at the Erasmus MC University Medical Center (academic hospital). Purposive sampling was used to obtain a variable sample of AD patients in terms of age, sex, and therapeutic experiences [14, 16, 17]. Eligible patients were recruited at the outpatient clinic of the Erasmus MC and Maasstad Hospital (general hospital, Rotterdam) and received a study information leaflet. Patients could sign up by contacting the researchers. They were offered a voucher of $€ 40$ for participating. After including three focus groups with a total of 20 participants (6-8 participants per focus group), we concluded that data saturation was reached and recruitment of participants ended.

\section{Data collection}

Prior to the start of the focus groups, patients provided written informed consent and completed a patient characteristics questionnaire. After we made sure that all participants felt able to share their views and experiences, the focus groups were started, structured by a topic guide (see Online Resource 1), which was based on researchers' experiences, literature, and therapy guidelines [18, 19]. Discussions were moderated by an experienced moderator of focus groups (ML or SE) and a physician with extensive experience in treating AD patients, but not involved in direct patient care of the participants (LW). All focus groups were audiotaped and transcribed verbatim.

\section{Data analysis}

The transcripts were analyzed by using Nvivo version 12 plus. An inductive approach to data analysis was applied using several phases of coding (guided by a codebook) combined with the constant comparison technique (comparing emerged concepts with new data and across groups) [20]. First, the transcripts were thoroughly read and roughly summarized by one researcher (LW) to get a holistic understanding of the focus groups. Subsequently, the first two transcripts were openly coded (i.e., inductively and line by line) [20] by one researcher (LW) and independently checked by another (ML). This resulted in a list of unstructured codes. More abstract and structured concepts of relevance were obtained through axial coding [20]. Using this structured coding scheme, the transcript of the third focus group was coded (LW) and independently checked (SE). No new concepts emerged during coding of the third focus group and data saturation was reached. The identified concepts and their subcategories were discussed during several multidisciplinary (psychologist, dermatologist and $\mathrm{PhD}$ student) research team meetings. This resulted in an overview of core needs and preferences within three central aspects of $\mathrm{AD}$ care (see Fig. 1).

\section{Results}

\section{Patient characteristics}

Table 1 and Online Resource 2 provide sample characteristics and demographics.

\section{Patients' needs and experiences in AD care: a patient-tailored approach}

Several needs and preferences were identified within three main aspects of AD care (Fig. 1): consultations with physicians, organization of $\mathrm{AD}$ care, and the therapeutic decisionmaking process. These needs and preferences are described in detail below. Additional illustrative quotes can be found in Online Resource 3. The need for a patient-tailored approach emerged as an overarching theme in all aspects of AD care. Patients stressed the need to better take patients' characteristics and personal needs and preferences into account throughout AD care.

\section{The consultations with physicians}

\section{Need for recognition of the physical and emotional impact of $A D$}

Patients reported that the major impact on both physical and emotional well-being of $\mathrm{AD}$ is sometimes underestimated by physicians. Patients emphasized the need for an increased 


\section{Patients' needs and preferences in atopic dermatitis care: a patient-tailored approach}
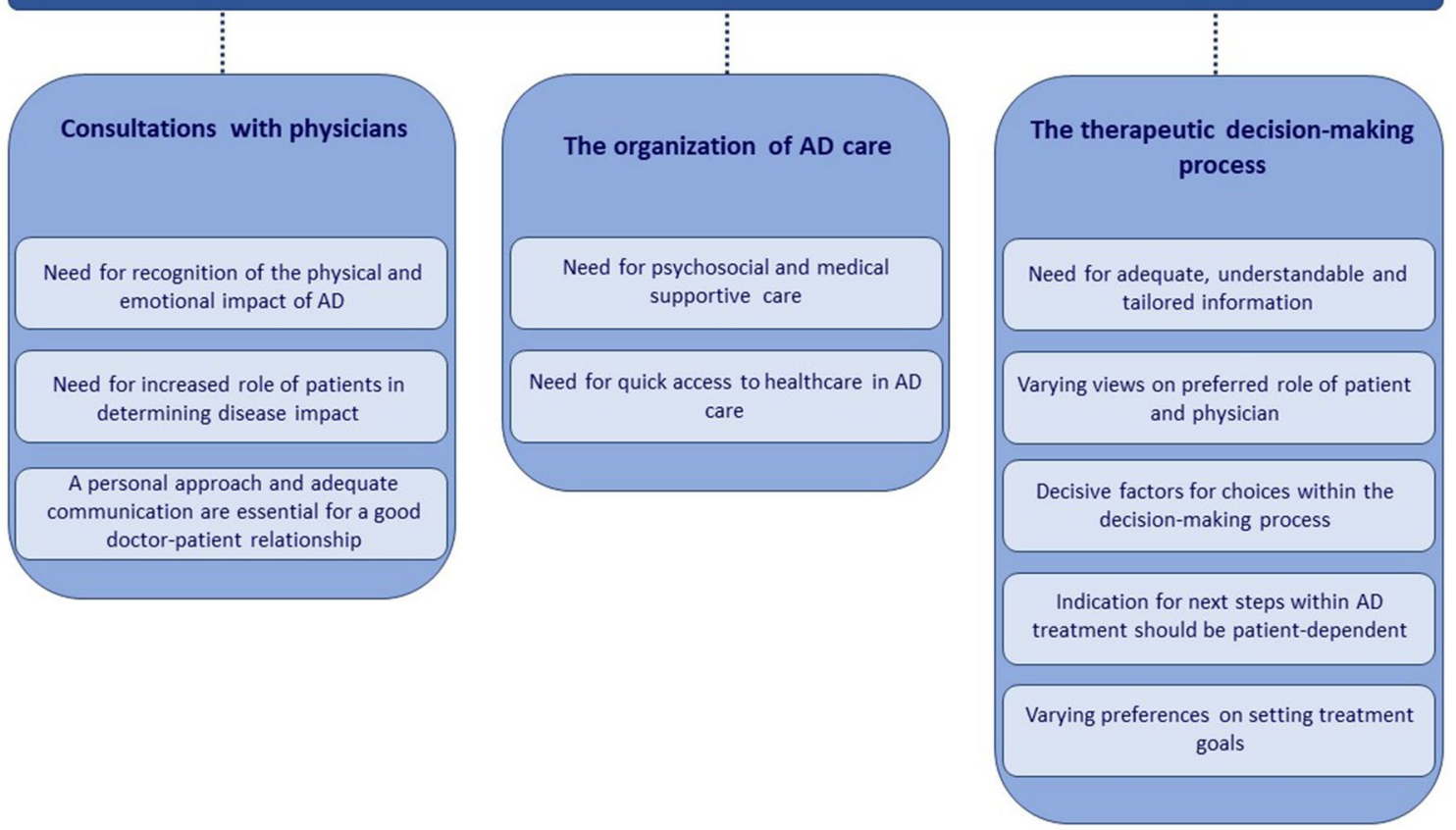

Fig. 1 Overview of needs and preferences of patients in AD care. Needs and preferences regarding several topics withing three main aspects of $\mathrm{AD}$ care were identified

Table 1 Participants' characteristics

\begin{tabular}{lllll}
\hline Setting & $\begin{array}{l}\text { Focus group 1 } \\
\text { Erasmus MC }\end{array}$ & $\begin{array}{l}\text { Focus group 2 } \\
\text { Maasstad Hospital }\end{array}$ & $\begin{array}{l}\text { Focus group 3 } \\
\text { Erasmus MC }\end{array}$ & Total \\
\hline $\begin{array}{l}\text { Participants— } n \\
\text { Age (years) }\end{array}$ & 8 & 6 & 6 & 20 \\
Median (IQR) & $34(20-48)$ & $28(27-57)$ & $33(31-40)$ & $31(24-48)$ \\
Min, max & 19,63 & 21,62 & 20,63 & 19,63 \\
Female— $n(\%)$ & $5(64)^{\mathrm{a}}$ & $4(67)$ & $2(33)$ & $11(55)$ \\
Current treatment—n $(\%)$ & & & $4(20)$ \\
Topical & $1(12.5)$ & $2(33)$ & $1(17)$ & $11(55)$ \\
Systemic immuno- & $5(63)$ & $4(67)$ & $2(33)$ & $5(25)$ \\
$\quad$ suppressant & $2(25)$ & $0(0)$ & $3(50)$ & $3(15)$ \\
Biologic & $1(12.5)$ & $0(0)$ & $2(33)$ & $2(10)$ \\
Self-reported impact of disease-n & & $0(0)$ & $3(15)$ \\
Very low & $2(25)$ & $0(0)$ & $0(0)$ & $4(20)$ \\
Low & $1(12.5)$ & $2(33)$ & $1(17)$ & $8(40)$ \\
Neutral & $2(25)$ & $1(17)$ & $3(50)$ & \\
High & $2(25)$ & &
\end{tabular}

$I Q R$ interquartile range

${ }^{\mathrm{a}} n=1$ transgender man 
recognition of the total burden and chronic pattern of their disease. Receiving empathy and being taken seriously were reported to be crucial in $\mathrm{AD}$ care.

'The moment you tell your physician that this is a real issue in your life, that it really is all-consuming, and your physician responds with "oh well, it's not that bad". It feels like a slap in the face.'- focus group (FG) 1

Patients identified a need for physicians to pay particular attention to certain feelings (e.g., shame, loneliness, stress, and fear) and behaviors (e.g., social avoidance and poor sleeping behavior) associated with AD. Patients prefer their physician to take the initiative in talking about the impact of the disease, as they often feel uncomfortable to do so themselves (e.g., because of the busy schedules of physicians).

\section{Need for increased role of patients in determining disease impact}

Patients mentioned that physicians should take into account that the consultation is only a snapshot, which complicates the assessment of a relapsing disease. Patients highlighted the need to be able to show the eczema at its worst, for which patients use tools including writing down symptoms or photographing lesions during exacerbations. However, when discussing the severity of their disease in times of remission, they often feel unheard.

'AD does not only feel like a struggle against your own body, but it is also a struggle to be taken seriously and to prove that you are eligible for certain therapies instead of being sent home with another ointment.' FG 3

Patients expressed the need for an increased role of patients in determining disease impact. Most patients agreed that the treating physician is responsible for the evaluation of the medical situation (e.g., giving instructions, discussing treatment options, and laboratory tests). However, atopic dermatitis does not stop when the patients leave the clinic and patients indicated that they themselves are responsible for evaluating the disease impact in daily life. As such, patients highlighted that a physician's respect for their autonomy in assessing the impact on their daily life would be appreciated.

\section{A personal approach and adequate communication are essential for a good doctor-patient relationship}

Patients indicated that the relationship between patient and physician plays an important role in their perceived quality of $\mathrm{AD}$ care. Patients prefer a personal approach and want their physician to be familiar with their situation, without making them feel like "one of many". Although patients prefer repeated consultations with the same physician, they believe a switch in physicians does not negatively influence the quality of care as long as the collaboration and communication between physicians is good. In terms of communication, patients stressed the importance of physicians who truly listen to their story and make them feel at ease.

'It feels so good that my physician takes time for me, because my experiences with previous physicians was like 'here are your ointments, good luck.' - FG 2

According to patients, simple signs, including eye contact, physicians introducing themselves properly at the first consultation, and asking for patients' preferences regarding $\mathrm{AD}$ care (i.e., ranging from how extensive information provision should be to the type of treatment they prefer) can facilitate in building an adequate relationship. Furthermore, patients indicated that it is very important that physicians adapt to the communicative level of the patient by, for instance, speaking in layman's terms, taking time to explain, and actively asking if they have any questions.

\section{The organization of AD care}

\section{Need for psychosocial and medical supportive care}

Patients indicated a need for increased and low-threshold psychosocial and medical supportive care. Patients understand that a physician's time is limited and accept that this additional care is provided by other health-care workers. Patients mentioned that initial supportive care by means of extra attention and time for questions could be provided by specialized nurses.

'A physician has limited time during a consultation, I do understand that, but sometimes you are feeling so bad and so itchy, and everything that comes with that. It is a real problem and feels like a handicap. In situations like this, an additional appointment with a nurse who has time to listen to your story, who is in close contact with your physician, and could potentially discuss any important considerations, would be really appreciated.' - FG2 
However, the need for more profound psychosocial support provided by a psychologist or therapist varied between patients. Patients indicated that this need should be identified during their consultations and that support should be easily available when needed. Patients experienced that getting an appointment with a psychologist often takes time and effort from their side. Therefore, a direct referral to a psychologist or therapist, in particular one with experience in skin diseases, would be highly appreciated. Furthermore, patients often reported positive experiences with support from patient associations and peer contact. As such, they felt it would be useful to provide extra information about these associations in hospitals and other health-care institutions since many patients might not be aware of the existence of these groups.

\section{Need for quick access to health care in AD care}

As reported by patients, AD care is not always easy accessible during disease flares. Patients experienced that timing of standard consultations is not always appropriate due to the fluctuation in severity over time. As such, they expressed the need for quick access to care when their disease flares, to optimize treatment and induce remission as soon as possible. They did not report a preference for a dermatologist o,r e.g., specialized nurse in particular. However, patients mentioned several complicating factors in this process; it is difficult for them to get in touch with their physician, and the nonmedical staff (e.g., administrative staff taking phone calls) is not able to understand or adequately assess the severity of their flares.

'My doctor tells me 'Do call us if it gets worse'. And when I do, the assistant tells me my doctor is not available, but that I can get consultation by phone. Well, that easily takes three days and by the time I can speak to my doctor my symptoms have reduced.' - FG 1

According to patients, clear instructions at the initial consultation on what to do in case of exacerbations, and an appropriate and personal doctor-patient relationship could facilitate quick access to care.

\section{The therapeutic decision-making process}

\section{Need for adequate, understandable, and tailored information}

Patients indicated that being provided with adequate and understandable information is crucial in AD care. This information should be patient tailored since some prefer comprehensive information, while others prefer a more concise overview.
Patients highlighted several aspects in the content of this information that were crucial to them. First, patients mentioned that they are more motivated to adhere to therapy when they are aware of the underlying principles. Therefore, patients require improved disease-related information. Second, patients would like to be informed about different treatment options, their mechanism of action, and possible side effects. This should preferably be tailored to the individual patient's situation and preferences. Additionally, patients preferred to be informed about potential next steps in the treatment process. They stated that knowing that there are other options available when necessary will put them at ease. Third, information about possible external influences on their disease such as the influence of nutrition, climate, and allergies as well as practical recommendations (e.g., daily care skin products) would be highly appreciated. On the other hand, patients indicated that repeating basic advices (e.g., advice for limited water exposure) is very bothersome, since they feel that they already "passed that stage", which emphasizes the need for continuous patienttailored information.

'I hear the same advice all the time: "you should not shower too often, not too warm and not too long; don't use soap". It is so annoying to hear this for the eighth time...'-FG 2

Patients believe that underexposure of specific topics (e.g., nutritional influences) in evidence-based guidelines used by physicians might be related to a lack of evidence. Even though patients understand that there might be a lack of scientific evidence in information about these topics on the Internet, patients would appreciate physicians to understand their search for additional information. In this context, an online forum monitored by physicians would be of added value. Meetings organized by patient associations were also often considered very informative. In addition, patients considered having the possibility to access their digital medical file a positive development.

\section{Varying views on the preferred role of patient and physician}

Patients vary in their views regarding the preferred role of the physician and patient in the decision-making process. Some patients preferred to have a leading role and want to be informed about different treatment options by their physician, resulting in an interactive decision-making process. According to these patients, getting enough time to read and consider the provided information is essential. This autonomy would enhance shared decision-making and 
reduce the "trial and error" feeling experienced by some patients.

'I prefer it to be an interaction between me and my physician, in which I also have a say. I know myself best and I read up on specific therapies.'-FG1

Other patients prefer their physicians to have the leading role since these patients feel that their physicians have a better overview of their situation and treatment options.

'I often feel like 'Oh well, you are the professional, so tell me what would be best.'-FG2

Generally, patients stated that physicians should get insight into each individual patient's preference regarding their preferred role, to make appropriate decisions for each patient.

\section{Decisive factors for choices within the decision-making process}

Patients mentioned that several factors play an important role in their therapeutic choices. Most patients indicated that they are willing to accept side effects as long as they are reversible and if the therapy shows sufficient effectiveness on their skin disease and quality of life. However, other patients feel resistance to "create new problems, by treating the initial problem'.

'I always weigh the options: it is either one thing or the other. If I want to get rid of my eczema, I need to accept certain side effects.'-FG1

Patients reported that the method and frequency of administration are important in terms of feasibility, and that this should be considered by physicians as well. Additionally, patients indicated that treatment choices are also dependent on previous therapeutic experiences. They considered the physician's recommendations and expectations regarding specific treatment options important factors as well.

Lastly, patients indicated that practical aspects including travel time and travel costs do not determine their therapeutic choices, as long as they receive high-quality care.

\section{Indication for next steps within AD treatment should be patient dependent}

Patients stated that a next step in their treatment is indicated when their current therapy is insufficiently effective (i.e., the disease negatively interferes with daily life) or inappropriate (e.g., due to side effects). Determining whether a certain new treatment is indicated should be patient dependent, as the impact of the disease in daily life and contributing factors differ between patients.
Most patients reported to know that therapeutic history is an important factor in their indication for new therapies (e.g., patients must have used at least 1 systemic immunosuppressant for 4 months to be eligible for dupilumab treatment [21]). Although patients have high expectations of novel therapies, they generally agree with the recommended stepwise approach when taking into account the high costs. However, they feel that physicians should be able to make some exceptions for very severe cases with a high impact on quality of life, although patients agreed that making these distinctions can be difficult.

'If someone is really suffering and they cannot even leave their bed and are not able to participate in normal life due to their eczema, then the physician should be able to skip a few steps in the treatment process.'FG 2

Additionally, patients voiced that when a patient has been treated with a systemic immunosuppressant without sufficient effectiveness, they should be able to step up more quickly the novel therapies. They stressed this could prevent a high burden for these patients.

\section{Varying preferences on setting treatment goals}

Most patients reported to have abstract treatment goals (e.g., increased quality of life), which are generally not discussed with their physician. Patients often feel uncertain about the effectiveness of the initiated therapy based on negative experiences with previous therapies. Consequently, they protect themselves from disappointment by not setting and discussing individual treatment goals.

'The physician can also not guarantee that my eczema will disappear completely. So setting big goals is an illusion for me because I find it difficult to know whether I am able to achieve that goal.'-FG2

Other patients mentioned that they experienced more guidance and motivation when setting and evaluating individual goals. Patients agreed that when realistic goals are discussed, subsequent evaluation of these goals during consultations is essential.

\section{Discussion}

This qualitative in-depth focus group study revealed the needs and preferences of patients regarding AD care in the era of new therapeutic options. A variety of needs and preferences were identified concerning consultations with physicians, AD care organization, and the therapeutic decision-making process. The need for a more patient-tailored 
approach was an overarching theme in all aspects of $\mathrm{AD}$ care.

With regard to consultations, patients stressed the need for autonomy in determining the impact of AD in daily life and increased recognition of the burden of disease by physicians [5]. To address these needs, supportive tools for patients to indicate the impact and disease control over a longer time period such as the Atopic Dermatitis Control Tool, validated health-related quality of life measures, or the use of personal health records (PHR) could be of added value [21-24]. Patients also indicated a need for psychosocial and medical supportive care. Studies assessing the implementation of supportive care for children with $\mathrm{AD}$ and their families have revealed positive results. Although applied interventions require further development, this could guide a more general implementation of supportive care [25-28].

Consistent with previous research [29, 30], our study showed that a trustful relationship with a personal approach, a physician who is truly listening to you, and the feeling of being taken seriously are essential for patients in $A D$ care. Ha et al. stated that many doctors overestimate their ability in communication, and therefore implementation of improved formal training in communication skills in the medical curriculum could be useful [29, 31]. A well-established doctor-patient relationship might also contribute to an increased accessibility in $\mathrm{AD}$ care during disease flares as physicians can often intuitively determine the urgency by knowing the patient personally. However, patients reported a struggle in contacting health-care providers when needed. Several solutions to enable quick access to health care have been described, including virtual care which has rapidly been adopted because of the COVID-19 pandemic [32]. Quick accessible care showed positive outcomes and optimized resource utilization in other chronic inflammatory conditions [33, 34]. Additionally, tools for self-management including PHR [24], flare self-assessment [35] and written eczema action plans with individualized guidance on, e.g., treatment use have been shown to be helpful in $\mathrm{AD}$ and other allergic diseases [36-40].

To create a therapeutic 'patient journey' that suits the patient best, the decision-making process should be patient tailored and optimized through, e.g., consideration of the patient's personal situation regarding eligibility and feasibility of therapies, and the patient's right to self-determination. Patients emphasized the importance of physicians being able to make patient-dependent exceptions on applied guidelines, which are based on relatively homogeneous populations. These exemptions are particularly needed when the disease highly interferes with patients' daily life. Previous studies have shown that therapeutic decisions should indeed be patient centered, fair, and cost-effective in the ideal situation [41]. However, this remains a practical challenge, partly because of the lack of insight into cost-effectiveness of the recently introduced therapies in daily practice [42]. A shift toward more patient-centered indication criteria incorporating the disease impact in daily life in addition to current criteria concerning therapeutic history would be highly appreciated by patients. This would facilitate in making "fair" choices, in particular for expensive therapies $[8,41]$. Agencies such as the FDA and the NHS also promote patient-centered care, with improved patient access to more affordable drugs [43-45]. In addition to patient-centered care (i.e., care that is respectful of, and responsive to, individual patient's preferences, needs, and values) [46], personalized medicine (i.e., tailoring care based on patients' genetic information or other biomarkers) [47] is also considered important to achieve individualized care [48]. Although their sources are different and it remains unclear whether and how these two could be combined on a practical level, personalized medicine might as well contribute to individual flexibility and variability in treatment decisions, and moving away from one-size-fits-all solutions [47].

In this study we investigated a contemporary topic using data obtained from a variable sample of patients, originating from a general and an academic hospital. Our qualitative data were analyzed using multiple phases of coding alternating with consensus discussions within our multidisciplinary team, enhancing the robustness of our results [20,49]. However, the design of this study does not allow us to draw conclusions on potential differences between patients from different medical settings. In addition to this study focusing on patients' views, future studies investigating views of healthcare providers on different aspects of AD care might be of added value in order to further optimize AD care.

This study demonstrated that AD patients have a variety of needs and preferences regarding care. AD care in general should be patient tailored, with increased attention for the psychosocial burden, as well as better access to healthcare during disease flares. To provide patient-tailored care, therapeutic decisions should be patient dependent and the interference of the disease in daily life should be incorporated when considering indication for novel therapies. Additional needs and preferences of patients concerning, for instance, the provided information or feasibility of therapies should be taken into account in the therapeutic decision-making process, with respect for the patient's autonomy.

Supplementary Information The online version contains supplementary material available at https://doi.org/10.1007/s00403-021-02321-z.

Acknowledgements The authors would like to thank the patients for participating in the focus groups and K.R. van Straalen for English language editing.

Author contributions All authors contributed to the study conception and design. Material preparation, data collection and analysis were 
performed by LWEMW, ML, SE, and DH. The first draft of the manuscript was written by LEMW and all authors commented on previous versions of the manuscript. All the authors read and approved the final manuscript.

Funding This study was multi-sponsored by Leo Pharma BV, Galderma and Janssen. None of the companies had involvement in the study design; collection, analysis and interpretation of data; in writing the report; nor in the decision to sbutmit the article for publication.

Availability of data and material, and code availability All data and materials as well as software application or custom code support their published claims and comply with field standards. The data that support the findings of this study are available from the corresponding author upon reasonable request.

\section{Declarations}

Conflict of interest The authors state no conflict of interest.

Ethics approval Our product-independent study was exempted from evaluation by the Medical Research Ethics Committee of the Erasmus MC (MEC-2019-0445).

\section{Consent to participate Not applicable.}

Consent for publication Patients signed informed consent regarding publishing their data.

Open Access This article is licensed under a Creative Commons Attribution 4.0 International License, which permits use, sharing, adaptation, distribution and reproduction in any medium or format, as long as you give appropriate credit to the original author(s) and the source, provide a link to the Creative Commons licence, and indicate if changes were made. The images or other third party material in this article are included in the article's Creative Commons licence, unless indicated otherwise in a credit line to the material. If material is not included in the article's Creative Commons licence and your intended use is not permitted by statutory regulation or exceeds the permitted use, you will need to obtain permission directly from the copyright holder. To view a copy of this licence, visit http://creativecommons.org/licenses/by/4.0/.

\section{References}

1. Kosse RC, Bouvy ML, Daanen M, de Vries TW, Koster ES (2018) Adolescents' perspectives on atopic dermatitis treatment-experiences, preferences, and beliefs. JAMA Dermatol 154(7):824-827

2. Howells L, Thomas KS, Sears AV et al (2019) Defining and measuring "eczema control": an international qualitative study to explore the views of those living with and treating atopic eczema. J Eur Acad Dermatol Venereol 33(6):1124-1132

3. Teasdale E, Muller I, Sivyer K et al (2020) Views and experiences of managing eczema: systematic review and thematic synthesis of qualitative studies. Br J Dermatol. https://doi.org/10.1111/bjd. 19299

4. Ring J, Zink A, Arents BWM et al (2019) Atopic eczema: burden of disease and individual suffering - results from a large EU study in adults. J Eur Acad Dermatol Venereol 33(7):1331-1340

5. Weidinger S, Novak N (2016) Atopic dermatitis. Lancet 387(10023):1109-1122

6. Wollenberg A, Barbarot S, Bieber T et al (2018) Consensusbased European guidelines for treatment of atopic eczema (atopic dermatitis) in adults and children: part II. J Eur Acad Dermatol Venereol 32(6):850-878

7. Lateef $F$ (2011) Patient expectations and the paradigm shift of care in emergency medicine. J Emerg Trauma Shock 4(2):163-167

8. Barry MJ, Edgman-Levitan S (2012) Shared decision making-pinnacle of patient-centered care. N Engl J Med 366(9):780-781

9. Ghio D, Muller I, Greenwell K et al (2020) "It's like the bad guy in a movie who just doesn't die": a qualitative exploration of young people's adaptation to eczema and implications for self-care. Br J Dermatol 182(1):112-118

10. Noerreslet M, Jemec GB, Traulsen JM (2009) Involuntary autonomy: patients' perceptions of physicians, conventional medicines and risks in the management of atopic dermatitis. Soc Sci Med 69(9): 1409-1415

11. Magin P, Adams J, Heading G, Pond D (2011) "Perfect skin", the media and patients with skin disease: a qualitative study of patients with acne, psoriasis and atopic eczema. Aust J Prim Health 17(2):181-185

12. Nelson PA, Thompson AR (2015) Judging quality in qualitative dermatology research: the science and the "art." Br J Dermatol 173(6):1351-1352

13. Krueger RA (2009) CMFGAPGfA, 4th edn. SAGE Publications, Thousand Oaks

14. Green J, Thorogood N (2018) Qualitative methods for health research, 4th edition ed: SAGE Publishing Ltd; 2018. Chapter 6, pp $147-172$

15. O'Brien BC, Harris IB, Beckman TJ, Reed DA, Cook DA (2014) Standards for reporting qualitative research: a synthesis of recommendations. Acad Med 89(9):1245-1251

16. Palinkas LA, Horwitz SM, Green CA, Wisdom JP, Duan N, Hoagwood K (2015) Purposeful sampling for qualitative data collection and analysis in mixed method implementation research. Adm Policy Ment Health 42(5):533-544

17. Green J, Thorogood N (2018) Qualitative methods for health research, 4th edition ed: SAGE Publishing Ltd. Chapter 3, pp $75-80$

18. NVDV (2018) Introductie van dupilumab voor ernstig constitutioneel eczeem (CE). www.nvdv.nl

19. richtlijn constitutioneel eczeem (2020) https://nvdv.nl/profession als/richtlijnen-en-onderzoek/richtlijnen/richtlijn-constitutioneeleczeem. Accessed 04 Apr 2020

20. Green J, Thorogood N (2018) Qualitative methods for health research, 4th edition ed: SAGE Publishing Ltd. Chapter 11, pp 285-311

21. Pariser DM, Simpson EL, Gadkari A et al (2020) Evaluating patient-perceived control of atopic dermatitis: design, validation, and scoring of the Atopic Dermatitis Control Tool (ADCT). Curr Med Res Opin 36(3):367-376

22. Apfelbacher CJ, Heinl D, Prinsen CA et al (2015) Measurement properties of adult quality-of-life measurement instruments for eczema: protocol for a systematic review. Syst Rev 4:48

23. Charman CR, Venn AJ, Williams HC (2004) The patient-oriented eczema measure: development and initial validation of a new tool for measuring atopic eczema severity from the patients' perspective. Arch Dermatol 140(12):1513-1519

24. Wells S, Rozenblum R, Park A, Dunn M, Bates DW (2014) Personal health records for patients with chronic disease: a major opportunity. Appl Clin Inform 5(2):416-429

25. Leong K, Ong TWY, Foong YW et al (2020) Multidisciplinary management of chronic atopic dermatitis in children and adolescents: a prospective pilot study. J Dermatolog Treat. https://doi. org/10.1080/09546634.2020.1782321

26. Ersser SJ, Cowdell F, Latter S et al (2014) Psychological and educational interventions for atopic eczema in children. Cochrane Database Syst Rev 2014(1):CD004054 
27. Ersser SJ, Farasat H, Jackson K, Dennis H, Sheppard ZA, More A (2013) A service evaluation of the Eczema Education Programme: an analysis of child, parent and service impact outcomes. Br J Dermatol 169(3):629-636

28. Jackson K, Ersser SJ, Dennis H, Farasat H, More A (2014) The Eczema Education Programme: intervention development and model feasibility. J Eur Acad Dermatol Venereol 28(7):949-956

29. Ha JF, Longnecker N (2010) Doctor-patient communication: a review. Ochsner J Spring 10(1):38-43

30. van Egmond S, Wakkee M, Droger M et al (2019) Needs and preferences of patients regarding basal cell carcinoma and cutaneous squamous cell carcinoma care: a qualitative focus group study. $\mathrm{Br}$ J Dermatol 180(1):122-129

31. Ranjan P, Kumari A, Chakrawarty A (2015) How can Doctors Improve their Communication Skills? J Clin Diagn Res 9(3):JE01-JE04

32. Wosik J, Fudim M, Cameron B et al (2020) Telehealth transformation: COVID-19 and the rise of virtual care. J Am Med Inform Assoc 27(6):957-962

33. Aziz A, Reynolds R, Bull C et al (2019) Flare to care-future management of long-term conditions. Future Healthc J 6(Suppl 1):85

34. Nene S, Gonczi L, Kurti Z et al (2020) Benefits of implementing a rapid access clinic in a high-volume inflammatory bowel disease center: access, resource utilization and outcomes. World J Gastroenterol 26(7):759-769

35. Danese S, Banerjee R, Cummings JF et al (2018) Consensus recommendations for patient-centered therapy in mild-to-moderate ulcerative colitis: the i Support Therapy-Access to Rapid Treatment (iSTART) approach. Intest Res 16(4):522-528

36. Zemek RL, Bhogal SK, Ducharme FM (2008) Systematic review of randomized controlled trials examining written action plans in children: What is the plan? Arch Pediatr Adolesc Med 162(2):157-163

37. Sauder MB, McEvoy A, Sampson M et al (2016) The effectiveness of written action plans in atopic dermatitis. Pediatr Dermatol 33(2):e151-153

38. Sauder MB, McEvoy A, Ramien ML (2016) Prescribing success: developing an integrated prescription and eczema action plan for atopic dermatitis. J Am Acad Dermatol 75(6):1281-1283
39. Powell K, Le Roux E, Banks JP, Ridd MJ (2018) Developing a written action plan for children with eczema: a qualitative study. Br J Gen Pract 68(667):e81-e89

40. van Os-Medendorp H, Deprez E, Maes N et al (2020) The role of the nurse in the care and management of patients with atopic dermatitis. BMC Nurs 19(1):102

41. Fleck LM, Danis M (2017) How should therapeutic decisions about expensive drugs be made in imperfect environments? AMA J Ethics 19(2):147-156

42. Zimmermann M, Rind D, Chapman R, Kumar V, Kahn S, Carlson J (2018) Economic evaluation of dupilumab for moderate-tosevere atopic dermatitis: a cost-utility analysis. J Drugs Dermatol 17(7):750-756

43. Gottlieb S. Statement from FDA Commissioner Scott Gottlieb, M.D., on new agency efforts to advance the patient voice in medical product development and FDA regulatory decision-making; 2018

44. Eaton S, Roberts S, Turner B (2015) Delivering person centred care in long term conditions. BMJ 350:h181

45. Roberts S, Eaton S, Finch T et al (2019) The Year of Care approach: developing a model and delivery programme for care and support planning in long term conditions within general practice. BMC Fam Pract 20(1):153

46. Institute of Medicine (US) Committee on Quality of Health Care in America (2001) Crossing the quality chasm; a new health system for the 21st century. National Academies Press (US), Washington (DC)

47. El-Alti L, Sandman L, Munthe C (2019) Person centered care and personalized medicine: irreconcilable opposites or potential companions? Health Care Anal 27(1):45-59

48. Chan IS, Ginsburg GS (2011) Personalized medicine: progress and promise. Annu Rev Genomics Hum Genet 12:217-244

49. Bazen A, Barg FK, Takeshita J (2021) Research techniques made simple: an introduction to qualitative research. J Invest Dermatol 141(2):241-247 (e241)

Publisher's Note Springer Nature remains neutral with regard to jurisdictional claims in published maps and institutional affiliations. 